\title{
A low cost wearable medical device for vital signs monitoring in low-resource settings
}

\author{
Muhammad Niswar', Muhammad Nur ${ }^{2}$, Amil A. Ilham ${ }^{3}$, Idar Mappangara ${ }^{4}$ \\ ${ }^{1,3}$ Department of Informatics, Faculty of Engineering, Universitas Hasanuddin, Indonesia \\ ${ }^{2}$ Department of Electrical Engineering, Faculty of Engineering, Universitas Hasanuddin, Indonesia \\ ${ }^{4}$ Faculty of Medicine, Universitas Hasanuddin, Indonesia
}

\begin{tabular}{l} 
Article Info \\
\hline Article history: \\
Received Jan 25, 2018 \\
Revised Jan 15, 2019 \\
Accepted Mar 9, 2019 \\
\hline
\end{tabular}

\section{Keywords:}

Biomedical sensors

IEEE802.11

Low-resource settings

Microcontroller

Vital signs

Wearable medical device

\begin{abstract}
Medical devices are often expensive, so people in low-income countries cannot afford them. This paper presents the design of a low-cost wearable medical device to measure vital signs of a patient including heart rate, blood oxygen saturation level $(\mathrm{SpO} 2)$ and respiratory rate. The wearable medical device mainly consists of a microcontroller and two biomedical sensors including airflow thermal sensor to measure respiratory rate and pulse oximeter sensor to measure $\mathrm{SpO} 2$ and heart rate. We can monitor the vital signs from a smartphone using a web browser through IEEE802.11 wireless connectivity to the wearable medical device. Furthermore, the wearable medical device requires simple management to operate; hence, it can be easily used. Performance evaluation results show that the designed wearable medical device works as good as a standard $\mathrm{SpO} 2$ device and it can measure the respiratory rate properly. The designed wearable medical device is inexpensive and appropriate for low-resource settings. Moreover, as its components are commonly available in the market, it easy to assembly and repair locally.
\end{abstract}

Copyright @ 2019 Institute of Advanced Engineering and Science. All rights reserved.

Corresponding Author:

Muhammad Niswar,

Department of Informatics, Faculty of Engineering,

Universitas Hasanuddin,

Jl. Poros Malino Km.6, Bontomarannu, Gowa 92172, South Sulawesi, Indonesia.

Email: niswar@unhas.ac.id

\section{INTRODUCTION}

The adoption of wearable medical devices for monitoring the health status becomes popular. The wearable health monitoring devices such as heart rate and blood oxygen saturation level $(\mathrm{SpO} 2)$ monitoring are commonly found in the market. However, there are many main barriers to access to medical device in low-resource settings including cost of medical device and lack of local production [1]. Many wearable medical devices in market are expensive, hence, most people in low-income countries could not afford to purchase them. Moreover, people cannot access to medical device due to lack of local production, which may cause by lack of electronic component supply chain. There are many medical devices designed for low-resources settings as described in reference [2] and this paper focuses on the design of a low-cost wearable medical device for monitoring vital signs including heart rate, $\mathrm{SpO} 2$ and respiratory rate that can be used for triaging the patient in hospital and home health care patient. We designed a wearable medical device suitable for low-resource settings and it can be locally assembled to increase access to medical devices. 


\section{RELATED WORKS}

Many researchers developed wearable medical devices for monitoring the vital signs such as electrocardiogram (ECG), SpO2 and pulse rate. Reference [3] developed a wearable medical system for monitoring vital signs including ECG, SpO2 and systolic blood pressure. Reference [4] designed a low-cost wearable wireless sensor device measures electrodermal activity and mobile plethysmography as well as discussing alternative wireless connectivity between sensor device and external computer such as mobile phone. Reference [5] developed a small-scale ECG monitoring device built with a heart rate monitor sensor and an Arduino microcontroller. Reference [6] designed a vital sign monitoring device that measures ECG, photoplethysmogram (PPG), and body temperature. Medical practitioner can remotely access the device to capture the sensing data through IEEE802.11 wireless connections. Reference [7] proposed a system that measures and monitors heart rate of patient. Reference [8] propose an ECG remote monitoring system that integrated to a broader Internet of Things (IoT) infrastructure. Reference [9] propose an ECG monitoring platform for system with minimal processing capabilities. Reference [10] propose a wireless ECG system using dry-contact electrodes. Reference [11] presents a low power ECG monitoring system using BMD101 sensor chip. Reference [12-16] developed a wearable medical device for measuring SpO2 and heart rate where Reference [16] developed a pulse oximeter for low resource settings.

Our designed wearable medical device is the improvement of our previous work [17] to meet the design considerations of a medical device for low-resource settings as described in Reference [18] in terms of cost, size, materials, and ease of manufacture. In our improvement design, we use IEEE 802.11 wireless standard for wireless connectivity, instead of using Bluetooth and ZigBee in our previous design. The reason behind the replacement is that the IEEE 802.11 standard is most commonly used for wireless connectivity and it can be easily connected to the internet, so that medical team can access the patient's vital signs data from anywhere using any mobile devices. The designed wearable medical device measures the vital signs of heart rate, $\mathrm{SpO} 2$ and respiratory rate and it can be used for triage in an emergency room and a disaster area.

\section{SYSTEM DESIGN}

Our wearable medical device mainly consists of biomedical sensors units, microcontroller, output unit, RF unit and power supply. Biomedical sensors include an airflow thermal sensor for respiratory rate measurement and pulse oximetry for heart rate and blood oxygen saturation measurement. RF unit consist of IEEE802.11 standards for wireless connection. There are two output units, i.e., a 1.3 inches OLED display and three LED indicators. The OLED displays heart rate and $\mathrm{SpO} 2$ status of a patient. The three LED indicators display three levels of patients' severity condition. Red LED indicates that patient has a major injury and need an immediate medical treatment. Yellow LED indicates that patient has a minor injury and treatment can be delayed for short period of time. Green LED indicates that patient in a normal condition. Figure 1 and 2 show the system block diagram and physical of the wearable medical device, respectively.

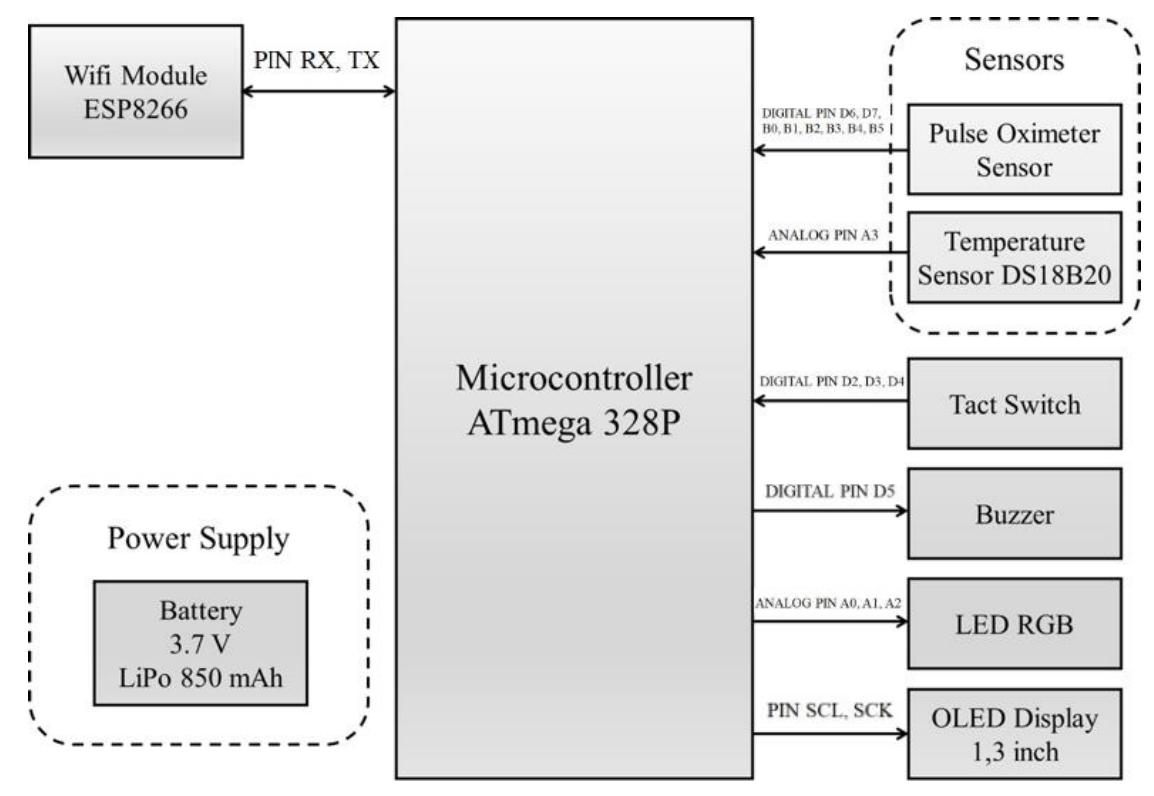

Figure 1. System block diagram 

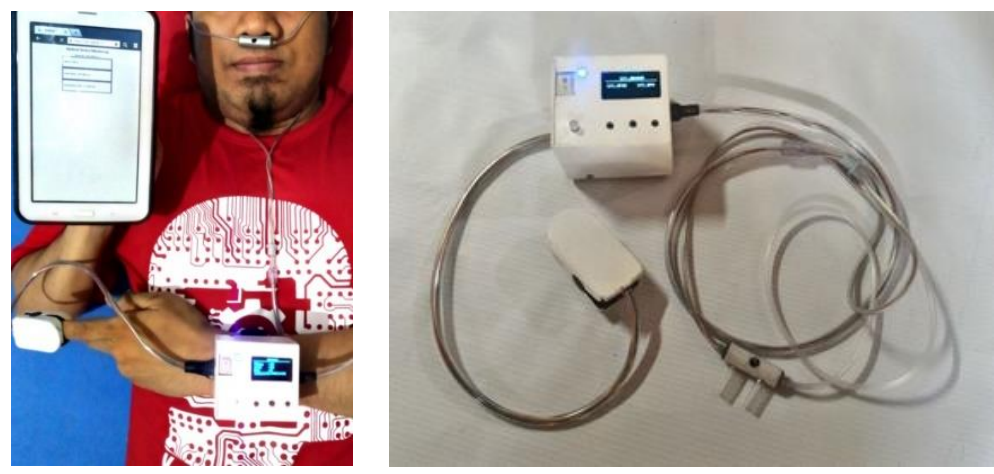

Figure 2. Wearable medical device

The device works as follow. First, the device measures the breath rate. If the breath rate is between 15 and 30 RPM, then the device measures the pulse rate and the percentage of SpO2. If the breath rate is between 10 and 14 RPM or 31 and 35 RPM, then the device alerts that patient is a minor condition by turning on the Yellow LED. Otherwise, the device turns on the Red LED indicating the major condition. If the pulse status is between 60 and $100 \mathrm{BPM}$, then the device measures the percentage of SpO2. If the pulse rate is between 50 and 59 BPM or 101 and 110 BPM, then the device turns on the Yellow LED indicating the minor condition. If the percentage of $\mathrm{SpO} 2$ is greater than 94\%, Green LED is turned on indicating that the patient is in normal condition. If the $\mathrm{SpO} 2$ is between $90 \%$ and $94 \%$, then the device turns on the Yellow LED indicating the minor condition. Otherwise, Red LED is turned on indicating the major condition. We embedded an algorithm of severity level classification based on vital signs condition in the microcontroller as shown in Algorithm 1.

Algorithm 1. Severity levels classification based on vital signs condition

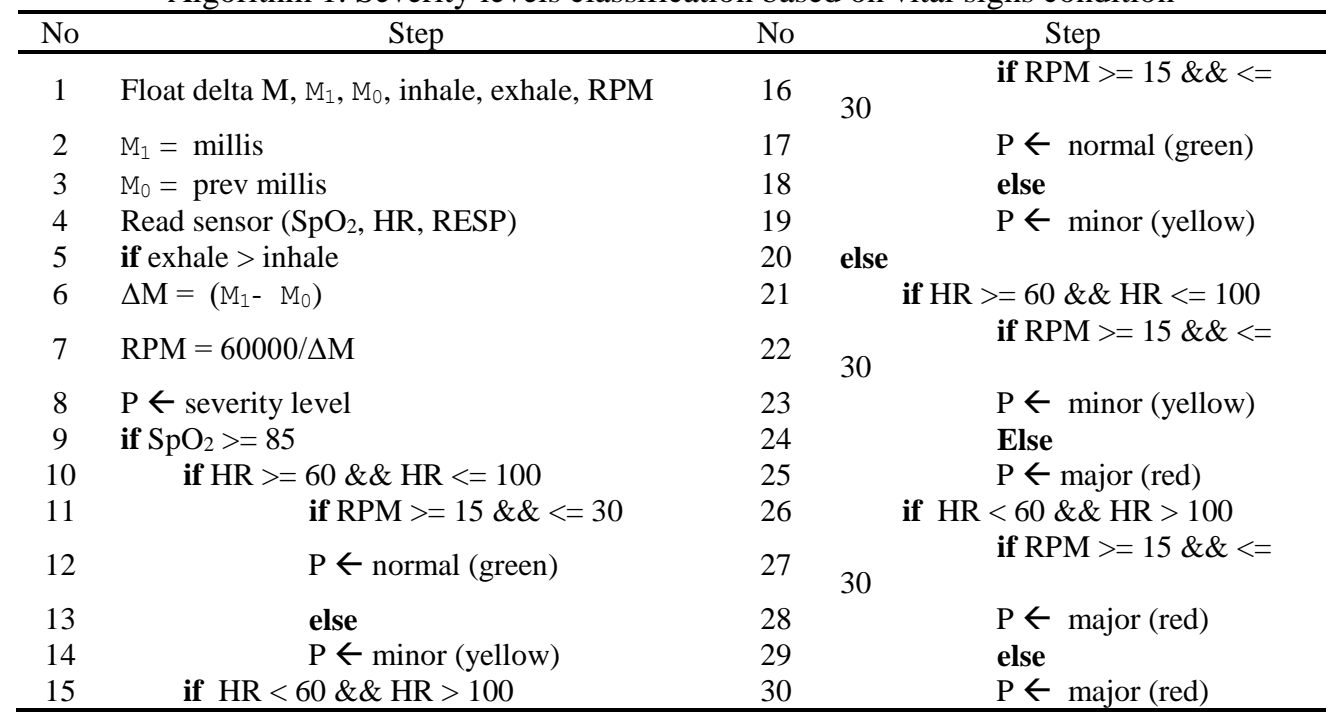

\section{BIOMEDICAL SENSORS}

The designed wearable medical device has two biomedical sensors, i.e., pulse oximeter and airflow thermal sensors. The pulse oximeter measures the level of blood-oxygen saturation of hemoglobin in the arterial blood ( $\mathrm{SpO} 2)$, and pulse rate. On the one hand, the airflow thermal sensor measures the respiratory rate. The following subsections describe in details of these two biomedical sensors.

\subsection{Pulse oximeter}

Pulse oximeter is a non-invasive device to measure human hemoglobin saturation and pulse rate through finger. It consists of a probe with two LEDs (red light and infrared light) and a light-detector. The blood and tissues absorb some of the light emitted by the probe. The light absorbed by the blood varies with the oxygen saturation of hemoglobin. The light detector measures the intensity of each light and

A low cost wearable medical device for vital signs monitoring in low-resource settings (Muhammad Niswar) 
microcontroller calculates the difference in the light absorption of $\mathrm{HbO}$ and hemoglobin without oxygen $(\mathrm{Hb})$ in the unit of percentage. The normal percentage of oxygen saturation is greater than $94 \%$. A pulse oximeter is inexpensive medical device and is priced as low as $\$ 10$ [19]. We customized a $\$ 20$ finger-type pulse oximeter to be integrated into our wearable medical device so that pulse oximetry data can be classified into three levels of patients' severity condition and transmitted to server or to mobile devices in both ad-hoc and infrastructure mode.

\subsection{Respiration sensor}

To measures the respiratory rate, we leverage a digital temperature sensor DS18B20 mounted in a nostril and measures the number of breaths that a patient takes each minute. Airflow that comes out from nose when exhaling contains $\mathrm{CO} 2$ that has a higher temperature than the airflow comes into the nose when inhaling. Therefore, the sensor can measure the number of exhaling per minute. To calculate the respiratory rate, we define the respiration period, i.e., the time difference between the last and previous time the patient is breathing and divided by sixty seconds. This DS18B20 measures temperature range from $-55^{\circ} \mathrm{C}$ to $125^{\circ} \mathrm{C}$ $\left(-67^{\circ} \mathrm{F}\right.$ to $\left.+257^{\circ} \mathrm{F}\right)$ and it is fairly precise $\left( \pm 0.5^{\circ} \mathrm{C}\right.$ Accuracy from $-10^{\circ} \mathrm{C}$ to $\left.+85^{\circ} \mathrm{C}\right)$ [20]. The price of the DS18B20 is approximately $\$ 2$ in the market. Device Technical Specification as shown in Table 1.

Table 1. Device technical specification

\begin{tabular}{ll}
\hline Components & Specification \\
\hline Microcontroller & ATMega328p \\
Clock Speed & $16 \mathrm{MHz}$ \\
Register Width & 8 -bit \\
RAM & $4 \mathrm{~KB}$ \\
Flash & $32 \mathrm{~KB}$ \\
Output Current & $184 \mathrm{~mA}$ \\
Output Power & $607 \mathrm{mV}$ \\
Sensors & Pulse Oximeter, Airflow Temperature Sensor (DS18B20) \\
Wireless Interface & IEEE802.11 (ESP8266 Chip) \\
Output Interface & Three LEDs, 1.3-inch OLED Display \\
Battery & Rechargeable LiPo 850 mAh \\
Operation Time & $3.2-4.1$ hours \\
Size & $6.5(\mathrm{~W})$ x 5.5(H) x 4(D) cm \\
Weight & 180 gr \\
\hline
\end{tabular}

\section{WIRELESS CONNECTIVITY FOR REMOTE MONITORING}

We use an ESP8266 Wi-Fi module [21] as a wireless connectivity for sending vital signs data to server and to mobile device in both ad-hoc and infrastructure mode as shown in Figure 3. The ESP8266 module is a low-cost chip that contains a system on-chip with TCP/IP protocol stack. The price of this module is approximately $\$ 5$, which is cheaper than the other Wi-Fi shield for embedded device. The module can be connected with any microcontroller through the serial UART using the standard AT command and can be programmed using Arduino IDE. Through the ESP8266, we can monitor the condition of patient remotely through a web browser in a smartphone as shown in Figure 4.

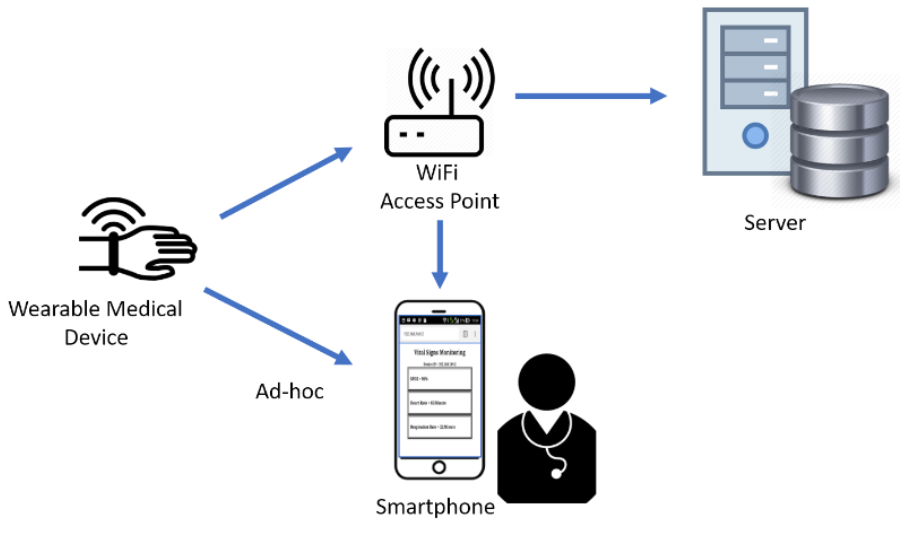

Figure 3. Network model

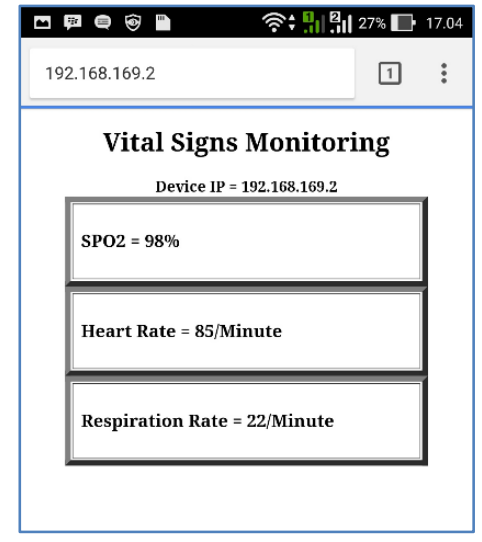

Figure 4. Vital signs monitoring via a browser in smartphone 


\section{PERFORMANCE EVALUATION}

We evaluate the performance of our designed wearable medical device in terms of biomedical sensors accuracy. We evaluate the accuracy of $\mathrm{SpO} 2$ and airflow thermal sensor by comparing it with standard $\mathrm{SpO} 2$ device and manual measurement, i.e., respiration is measured adhoc.

\subsection{Evaluation of $\mathrm{SpO} 2$ pulse oximeter}

We conduct a comparative study of our designed medical device with standard $\mathrm{SpO} 2$ of Nellcor OxiMax in Penlon SP M5. The aim of this study is to evaluate SpO2 accuracy performance of our designed medical device under non-motion condition. We leverage ProSim SPOT Light SpO2 Pulse Oximeter Analyzer to test the functionality of our designed medical device. This pulse oximetry analyzer can generate the physiological signal of interest such as various values of saturation and heart rate to mimic the patient condition. We applied this device to our designed medical device and to Nellcor OxiMax $\mathrm{SpO} 2$. We set the ProSim SPOT Light SpO2 to generate the signal SpO2 saturation of 70, 75, 80, 85, 90, 95, 97, 98 and 100 and heart rate of $30,60,65,80,100,120,150,180$ and 240. We use the Pearson correlation coefficient statistical analysis to describe the correlation between our designed medical device and standard $\mathrm{SpO} 2$ of Nellcor OxiMax. Figure 5 shows the strong correlation between designed medical device and Nellcor OxiMax SpO2 indicating that our designed wearable medical device works as good as the standard $\mathrm{SpO} 2$ device.

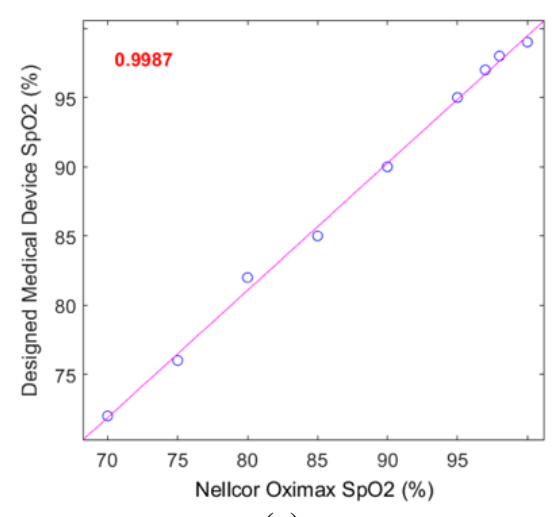

(a)

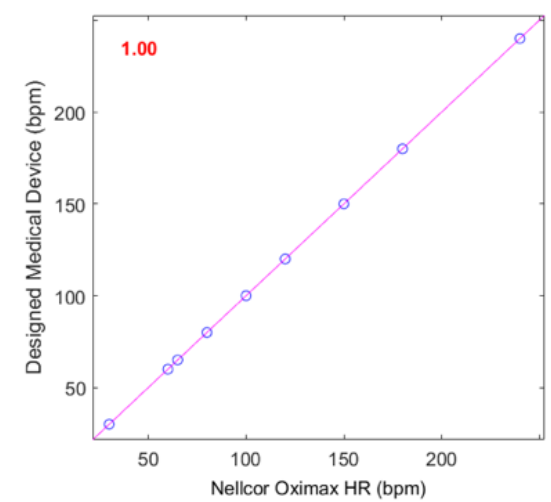

(b)

Figure 5. Correlation between designed medical device and Nellcor OxiMax SpO2,

(a) Oxygen saturation, (b) Heart rate

\subsection{Evaluation of respiration sensor}

The evaluation was conducted by monitoring the output temperature while the device was attached to the nose of patient. Figure 6 shows that the temperatures changes from $32^{\circ} \mathrm{C}$ to $32.25^{\circ} \mathrm{C}$. The temperatures goes to $32^{\circ} \mathrm{C}$ when patient is inhaling and it goes to $32.25^{\circ} \mathrm{C}$ when patient is exhaling. Some spikes occurs due to some movements of patient but the sensor can clearly distiguish temperature changes between inhaling and exhaling. The system counts the number of breath when patient is exhaling and Figure 6 shows that the number of breath is 14 times per minutes, i.e., the patient has abnormal respiration.

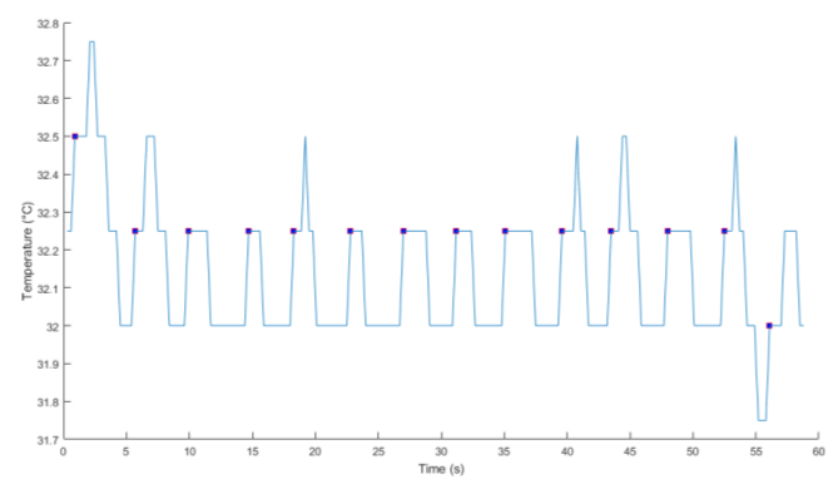

Figure 6. Temperature change in airflow thermal sensor

A low cost wearable medical device for vital signs monitoring in low-resource settings (Muhammad Niswar) 


\section{CONCLUSION}

This research developed a low-cost wearable medical device measuring the vital sign of patient. The designed wearable medical device is mainly built with two biomedical sensors (airflow thermal sensor and pulse oximeter sensor), a microcontroller (ATMEGA 328p), a wireless module (IEEE802.11) and a power supply, which are inexpensive and mostly available in the market. It measures heart rate, SpO2 level, and respiratory rate of patient and determine the severity level of patient based on vital signs condition. We use a ESP8266 Wi-Fi module for wireless connectivity to allow vital signs acquisition from the wearable medical device to smartphone for remote monitoring. We evaluated the performance of the designed device by comparing with the standard SpO2 of Nellcor OxiMax in Penlon SP M5. The result shows that the designed wearable medical device works as good as standard $\mathrm{SpO} 2$ device and it can measure the respiratory rate properly.

\section{ACKNOWLEDGEMENTS}

The authors would like to thank the teaching hospital Universitas Hasanuddin for supporting this research by providing access to their medical devices to be compared to our designed wearable medical device.

\section{REFERENCES}

[1] Adriana Velazquez Berumen, "Need for appropriate, Affordable, and Safe Medical Devices," 11th Annual University of Minnesota Design of Medical Device (DMD) Conference, 2012.

[2] World Health Organization, "WHO Compendium of innovative health technologies for low resource settings," 2015.

[3] T. Gao et al., "The Advanced Health and Disaster Aid Network: A Light-Weight Wireless Medical System for Triage," in IEEE Transactions on Biomedical Circuits and Systems, vol. 1, no. 3, pp. 203-216, Sept. 2007.

[4] R. R. Fletcher, M. Poh and H. Eydgahi, "Wearable sensors: Opportunities and challenges for low-cost health care," 2010 Annual International Conference of the IEEE Engineering in Medicine and Biology, Buenos Aires, pp. 1763-1766, 2010.

[5] Kristine Joyce P. Ortiz, John Peter O. Davalos, Elora S. Eusebio, Dominic M. Tucay, “ IoT: Electrocardiogram (ECG) Monitoring System, “ Indonesian Journal of Electrical Engineering and Computer Science Vol. 10, No. 2, pp. 480 489, May 2018.

[6] Achmad Rizal, Vera Suryani, Jondri, Sugondo Hadiyoso, “ Development of Wireless Patient's Vital Sign Monitor Using Wireless LAN (IEEE.802.11.b/g) Protocol,” International Journal of Electrical and Computer Engineering (IJECE), Vol. 4, No. 6, pp. 893 901, December 2014.

[7] Nabeel Salih Ali, Zaid Abdi Alkaream Alyasseri, Abdulhussein Abdulmohson, " Real-Time Heart Pulse Monitoring Technique Using Wireless Sensor Network and Mobile Application," International Journal of Electrical and Computer Engineering (IJECE), Vol.8, No.6, pp. 5118 5126, December 2018.

[8] E. Spanò, S. Di Pascoli and G. Iannaccone, "Low-Power Wearable ECG Monitoring System for Multiple-Patient Remote Monitoring," in IEEE Sensors Journal, vol. 16, no. 13, pp. 5452-5462, July1, 2016.

[9] S. P. Preejith, R. Dhinesh, J. Joseph and M. Sivaprakasam, "Wearable ECG platform for continuous cardiac monitoring," 2016 38th Annual International Conference of the IEEE Engineering in Medicine and Biology Society (EMBC), Orlando, FL, pp. 623-626, 2016.

[10] E. Valchinov, A. Antoniou, K. Rotas and N. Pallikarakis, "Wearable ECG system for health and sports monitoring," 2014 4th International Conference on Wireless Mobile Communication and Healthcare Transforming Healthcare Through Innovations in Mobile and Wireless Technologies (MOBIHEALTH), Athens, pp. 63-66, 2014.

[11] Z. Ai, L. Zheng, H. Qi and W. Cui, "Low-Power Wireless Wearable ECG Monitoring System Based on BMD101," 2018 37th Chinese Control Conference (CCC), Wuhan, pp. 7374-7379, 2018.

[12] R. R. Adiputra, S. Hadiyoso, Y. Sun Hariyani, “ Internet of Things: Low Cost and Wearable SpO2 Device for Health Monitoring," International Journal of Electrical and Computer Engineering (IJECE), Vol. 8, No. 2, pp. 939 945, April 2018.

[13] N. Sutar, M. Parihar, R. Ijare and K. Gowari, "Design and development of SMD based wearable pulse oximeter," 2016 International Conference on Communication and Signal Processing (ICCSP), Melmaruvathur, pp. 1812-1816, 2016.

[14] Y. Xie, Y. Gao, Y. Li, Y. Lu and W. Li, "Development of Wearable Pulse Oximeter Based on Internet of Things and Signal Processing Techniques," 2017 European Modelling Symposium (EMS), Manchester, pp. 249-254, 2017.

[15] N. Watthanawisuth, T. Lomas, A. Wisitsoraat and A. Tuantranont, "Wireless wearable pulse oximeter for health monitoring using ZigBee wireless sensor network," ECTI-CON2010: The 2010 ECTI International Confernce on Electrical Engineering/Electronics, Computer, Telecommunications and Information Technology, Chiang Mai, pp. 575-579, 2010. 
[16] Z. Jones, E. Woods, D. Nielson and S. V. Mahadevan, "Design of a pulse oximeter for price sensitive emerging markets," 2010 Annual International Conference of the IEEE Engineering in Medicine and Biology, Buenos Aires, pp. 1085-1088, 2010.

[17] M. Niswar et al., "The Design of Wearable Medical Device for Triaging Disaster Casualties in Developing Countries," 2015 Fifth International Conference on Digital Information Processing and Communications (ICDIPC), Sierre, pp. 207-212, 2015.

[18] A. J. Nimunkar, J. Baran, D. Van Sickle, N. K. Pagidimarry and J. G. Webster, "Medical devices for developing countries: Design constraints and approaches," 2009 Annual International Conference of the IEEE Engineering in Medicine and Biology Society, Minneapolis, MN, pp. 7048-7051, 2009.

[19] World Health Organization, "Global Pulse Oximetry Project," First International Consultation Meeting WHO Headquarters, Geneva, Switzerland, 29-30th October 2008.

[20] Maxim Integrated, "Programmable Resolution 1-Wire Digital Thermometer" DS18B20 Datasheet [Revised Sept. 2018].

[21] Espressive Systems, “Espressive Smart Connectivity: ESP8266,” ESP8266 Datasheet, Oct.12, 2013. 